\title{
Footshock-produced excitation and inhibition of activity in rats*
}

\author{
HYMIE ANISMAN and T. GARY WALLER \\ University of Waterloo, Waterloo, Ontario, Canada
}

\begin{abstract}
Hooded rats received five 1 -sec shock presentations of $0.3,0.5$. or $1.0 \mathrm{~mA}$. Activity measures recorded during each 30 -sec intershock interval and at various retention intervals $(.10,2.5$, and $24 \mathrm{~h}$ ) following footshock (FS) indicated that (a) 5 sec after FS activity is directly related to shock intensity, while 10-30 sec following FS activity is inversely related to shock intensity; (b) the rate of decline in activity following FS increases with successive shock presentations; (c) activity is greater $10 \mathrm{~h}$ after $\mathrm{FS}$ than at a 2.5 - or $24 \mathrm{~h}$ retention interval; and finally (d) shock compartment confinement increased activity and resulted in a substantial alteration in the form of retention curve in the $0.3-\mathrm{mA}$ group but had lesser effects upon the retention curves of the $0.5-$ and 1.0-mA groups. The data were interpreted as supporting the hypothesis that the "incubation effect" is a result of a decline of the activating effects of FS.
\end{abstract}

It has been demonstrated that. at an intermediate retention interval, passive avoidance performance is superior to that demonstrated at a brief or long retention interval (Zammit-Montebello, Black. Marquis, \& Suboski. 1969). Several investigators (e.g., Anisman, 1972: Anisman \& Waller, 1971; Barrett, Leith, \& Ray, 1971) have ascribed these changes in avoidance performance to nonassociative changes in activity over time following shock. Specifically, it is assumed that footshock (FS) has a dual effect upon activity which need not be dependent on changes in fear level. That is, in addition to an inhibitory effect. FS also preduces a short-lived excitation which can last as long as $10 \mathrm{~min}$ (Pinel. Corcoran, \& Malsbury, 1971). Accordingly, activity levels may be a function of the interaction between the transient activating effects and the longer-lasting inhibitory effects of FS.

Given these earlier findings, one purpose of the present investigation was to determine whether (a) the FS-produced excitation is greater following high than low shock intensities; (b) the decline in FS-produced excitation. and the extent of the FS-produced immobility. is affected by shock intensity: and (c) the FS-produced activation and its subsequent decline are affected by repeated shock presentations. The present investigation was further designed to determine whether activity is lower at an intermediate than at either a brief or long retention interval and whether increases in the strength of the immobility response produced by high shock intensities (Blanchard \& Blanchard. 1970) or decreases in the strength of the immobility response through shock compartment confinement (Baun. 1970) alter the form of the nonmonotonic changes in activity seen at various intervals after shock.

*The research and preparation of this paper were supported by Grant A0-326 from the National Research Council of Canada to the second author. Requests for reprints should be addressed to Hymie Anisman. Department of Psychology. Liniversity of Waterloo, Waterloo, Ontario, Canada.

\section{METHOD}

\section{Subjects}

The Ss were 180 experimentally naive male hooded rats, weighing $230-270 \mathrm{~g}$, procured from the Canadian Breeding Laboratories. Rats were individually housed in standard wire cages and permitted ad lib access to food and water for the duration of the experiment.

\section{Apparatus}

The test chamber consisted of a wooden box, $71.2 \times 71.2 \times$ $60 \mathrm{~cm}$, with a grid floor made of $0.31-\mathrm{cm}$ brass rods spaced $1.27 \mathrm{~cm}$ apart (center to center). The chamber could be divided into two equal-sized compartments by the insertion of a plywood wall. The walls of one of the resulting compartments were covered with black Bristol board and those of the other with white Bristol board. The grid floor of the apparatus was wired to a shock generator and could deliver a 1-sec pulse of 0.3 . 0.5 . or $1.0 \mathrm{~mA}$ of shock (constant current, ac).

\section{Procedure}

\section{Training}

Rats were placed, one at a time, in the shock compartment for a 3-min period during which three independent groups received five unsignaled $1-\mathrm{sec}$ shock presentations of $0.3,0.5$, or $1.0 \mathrm{~mA}$ at 30 -sec intervals. One-half of the rats in each of the groups were shocked in the white compartment. while the remaining rats were shocked in the black compartment. Throlghout the training phase, movement ratings were recorded during intershock intervals. The rating procedure was modified from Anisman and Waller (1972) and essentially consisted of recording behavior on a 5 -sec time sampling basis. Movement was rated on a 4-point scale as described by Anisman and Waller (1972). Following shock exposure. rats were returned to their home aages.

\section{Retention Test}

Following one of three retention intervals $1.10,2.5$, or $24 \mathrm{~h}$ ), rits were returned to the open field (with the dividing partition removed) and placed in the nonshock side of the chamber for $5 \mathrm{~min}$. Activity was again recorded using a 10 -sec, rather than a 5-sec, time sampling procedure. In addition, number of crossings 


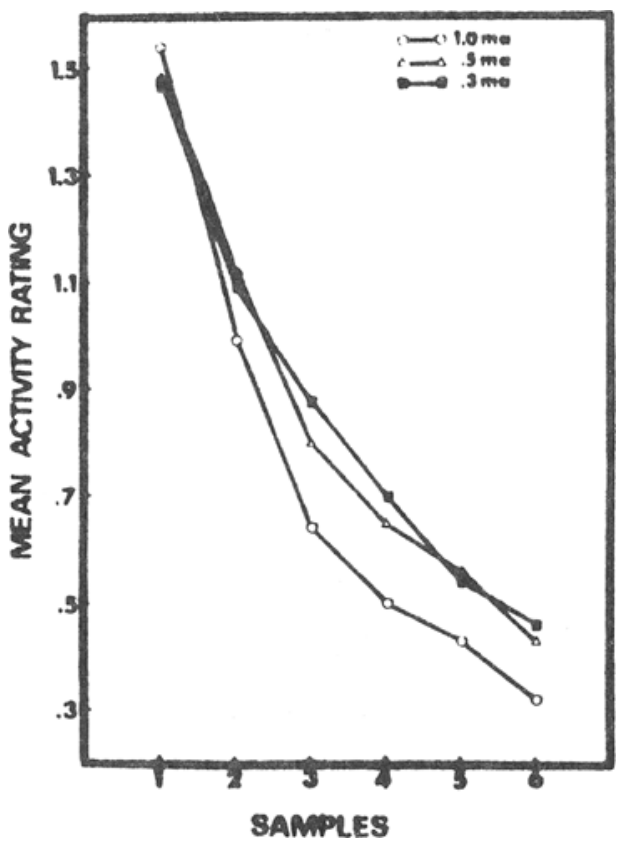

Fig. 1. Mean aetivity ratings during six successive 5-sec behavior samples as a function of shock intensity.

from the dangerous to the safe side were recorded for each rat. Half the rats in each of the nine groups received shock compartment confinement for $5 \mathrm{~min}$ immediately prior to the activity test. While the remaining rats spent an equivalent amount of time in their home cages. All rats were equated for amount of handling. Shock compartment confinement consisted of rats being returned to the shock compartment. in the absence of shock, without the availability of escape from that compartment. However, the rats were permitted free exploration of the shock compartment during the confinement period.

\section{RESULTS}

\section{Activity During Intershock Intervals}

Figure 1 shows the mean activity ratings for the $0.3-$, $0.5-$. and $1.0-\mathrm{mA}$ groups over each of the six sampling periods (collapsed over shock presentations). An analysis of variance of the activity ratings yielded a significant Shock Intensity by Samples interaction $(\mathrm{F}=2.68 \mathrm{df}=$ $10 / 4425, p<.05$ ). Subsequent Duncan's multiple-range tests on the simple main effects for the interaction indicated that activity declined among all groups during the 30-sec postshock period. The decline, as seen in Fig. 1. was more rapid in the $1.0-\mathrm{mA}$ group than in the 0.3 . and $0.5 \cdot \mathrm{mA}$ groups. In fact, $5 \mathrm{sec}$ after shock exposure, activity was greater in the 1.0- than in the 0.3 and $0.5-\mathrm{mA}$ groups, whereas $10-30 \mathrm{sec}$ after shock, the 1.0-mA group exhibited a lower level of activity than the remaining groups. Moreover, this trend was found to occur following four of the five shock presentations. When activity was collapsed over samples, the $1.0-\mathrm{mA}$ group was less active than the $0.3-$ and $0.5 \cdot \mathrm{mA}$ groups following the first and second shock presentations, while no differences occurred following the remaining three shocks (Intensity by Shock Presentations interaction, F $=2.80, \mathrm{df}=8 / 885, \mathrm{p}<.05)$.

The analysis of variance further revealed a significant Shock Presentations by Samples interaction $(F=4.09$, $\mathrm{df}=20 / 4425, \mathrm{p}<.01$ ). Multiple comparisons performed on the simple main effects involved in the interaction indicated that with successive shock presentations, the rate of decline in activity increased. In fact, the minimum activity level was noted to be a decreasing function of number of shock presentations. In contrast, the level of activity exhibited $5 \mathrm{sec}$ after shock (collapsed over shock intensity) did not vary with repeated exposure to shock.

\section{Retention}

As a result of the low level of activity exhibited by all groups, very few crossings were made from one chamber to the next. The analysis of number of crossings did not approach significance for any of the main effects or interactions. Figure 2 shows the mean total activity ratings for each of the three shock groups as a function of retention interval and confinement condition. The analysis of variance of the activity ratings yielded a significant main effect for retention interval $(F=4.69$. $\mathrm{df}=2 / 162, \mathrm{p}<.05$ ). Multiple comparisons on the means involved indicated that the 10 -h group was more active than the 2.5- and 24-h groups. Although the analysis of

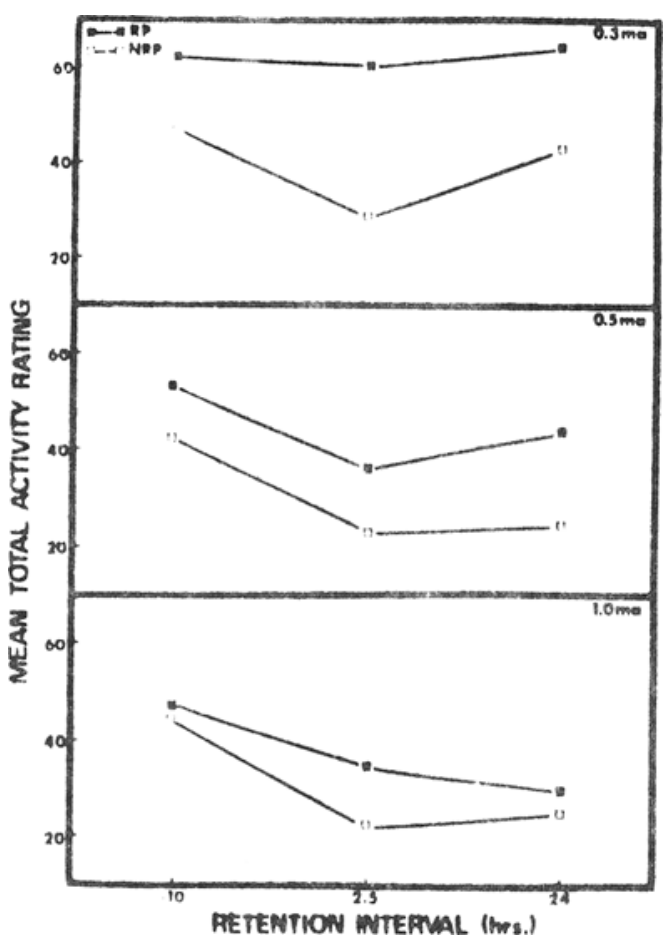

Fig. 2. Mean total activity ratings as a function of shock intensity, retention interval, and confinement condition, i.e. response prevention (RP) vs no response prevention (NRP). 
variance did not yield a significant Retention Interval by Shock Intensity interaction, multiple comparisons were carried out, since an a priori prediction had been made concerning this interaction (Winer. 1962). It was found that activity declined between .10 and $2.5 \mathrm{~h}$ in all groups $(\mathrm{p}<.05)$. Among rats in the $0.3-\mathrm{mA}$ group, activity increased between 2.5 and $24 \mathrm{~h}$ following shock $(p<.10)$. The differences in activity at these intervals did not approach significance in the $0.5-$ and $1.0 \mathrm{~mA}$ groups.

The analysis further yielded a significant Confinement by Shock Intensity interaction $(\mathrm{F}=3.53$, df $=2 / 162$, $\mathrm{p}<.05)$. Subsequent multiple comparisons on the simple main effects involved in this interaction indicated that, while shock compartment confinement increased activity in each group, the increase was greater among rats in the 0.3 - than the $0.5-\mathrm{mA}$ group. which in turn was greater than that of the $1.0-\mathrm{mA}$ group. The increase in activity among the confinement groups resulted in the eradication of the U-shaped function in the $0.3-\mathrm{mA}$ group, a progression towards a U-shaped function in the $0.5-\mathrm{mA}$ group, but little change in the form of the retention curve in rats shocked with $1 \mathrm{~mA}$.

\section{DISCUSSION}

With respect to the changes in activity seen immediately after shock, it is clear that (a) FS has both an excitatory and inhibitory effect on general activity, (b) the magnitude of the excitation is directly related to shock intensity, (c) the rate of decline of the FS-produced excitation as well as the strength of the FS-produced immobility are directly related to shock intensity and number of repeated shock presentations, and (d) although activity did decrease with repeated shock presentations. there was no decrease in activity $5 \mathrm{sec}$ after shock. One possible explanation which would account for the dual nature of shock on activity is the release of some chemical, which in turn triggers an antagonistic rebound. Recently, several investigators (Anisman, 1973; Pinel et al, 1971) suggested that the adrenergic and cholinergic systems might suit such a model. Specifically, soon after shock there is a sympathetic (adrenergic) reaction followed by a parasympathetic (cholinergic) rebound. Thus, it is the activation of the adrenergic system which produces the excitation, while activation of the cholinergic system results in the suppression of ongoing behavior.

Analyses of activity at the various retention intervals lend support to an activity explanation of the U-shaped performance function. Specifically. when freezing was moderate (1.e., 0.3-mA shock). allowing for both the descending and ascending portions of the Kamin effect. response prevention increased activity sufficiently to completely eradicate the U-shaped function. In contrast. when freezing was moderate $(0.5-\mathrm{mA}$ shock), such that activity did not increase between 2.5 and $24 \mathrm{~h}$, shock compartment confinement reduced freezing and consequently produced a retention curve which approximates a U-shaped function. If freezing was extremely intense, as in the 1.0-mA group, shock compartment confinement had negligible effects. It is possible, however. that with more response prevention, freezing would have been further reduced (Baum, 1970) and a $\mathrm{U}$-shaped function would have been observed in the $1.0 \mathrm{~mA}$ group. Taken together, the data of the present study suggest that the nonmonotonic change in avoidance performance following original shock exposure is a function of changes in the strength of the immobility response. Moreover, it is not unlikely that these changes are a result of the interaction between the excitatory and inhibitory effects of FS.

\section{REFERENCES}

Anisman, $H$. Cholinergic mechanisms and alterations in behavioral suppression as factors producing time dependent changes in avoidance performance. Journal of Comparative \& Physiological Psychology, 1973, in press.

Anisman, H., \& Waller. T. G. Effects of conflicting response requirements and shock compartment confinement on the Kamin effect in rats. Journal of Comparative \& Physiological Psychology, 1971, 77, 240-244.

Anisman, H., \& Waller. T. G. Facilitative and disruptive effects of prior exposure to shock on subsequent avoidance performance. Journal of Comparative \& Physiological Psychology, 1972, 78, 113-122.

Barrett, R. J., Leith. N. L., \& Ray, O. S. The Kamin effect in rats: An index of memory or shock induced inhibition. Journal of Comparative \& Physiological Psychology, 1971. 77. 234-239.

Baum, M. Extinction of avoidance responding through response prevention (flooding). Psychological Bulletin, 1970, 74. $276-284$.

Blanchard, R. J.. \& Blanchard, D. C. Crouching as an index of fear. Journal of Comparative \& Physiological Psychology. $1969,67,370-375$.

Pinel, J. P. J., Corcoran. M. E., \& Malsbury, C. W. The incubation effect in rats: A decline of foot-shock-produced activation. Journal of Comparative \& Physiological Psychology. 1971.77. 271-276.

Winer, B. J. Statistical principles in experimental design. New York: MiGraw-Hill. 1962.

Zammit-Montebello, A.. Black, M., Marquis, H. A., \& Suboski. M. D. Incubation of passive avoidance in rats: Shock intensity and pretraining. Journal of Comparative \& Physiological Psychology, 1969.69.579-582.

(Received for publicution August 18, 1972; icvision received October 23,1972 .) 